\title{
Solvability of some classes of singular integral equations of convolution type via Riemann-Hilbert problem
}

Pingrun $\mathrm{Li}^{1 *}$

\author{
"Correspondence: \\ lipingrun@163.com \\ 'School of Mathematical Science, \\ Qufu Normal University, Qufu, \\ P.R. China
}

\begin{abstract}
In this paper, we study methods of solution for some kinds of convolution type singular integral equations with Cauchy kernel. By means of the classical boundary value problems for analytic functions and of the theory of complex analysis, we deal with the necessary and sufficient conditions of solvability and obtain the general solutions and the conditions of solvability for such equations. All cases as regards the index of the coefficients in the equations are considered in detail. Especially, we discuss some properties of the solutions at the nodes. This paper will be of great significance for the study of improving and developing complex analysis, integral equation and boundary value problems for analytic functions (that is, Riemann-Hilbert problems). Therefore, the classical theory of integral equations is extended.
\end{abstract}

MSC: 45E05; 45E10; 30E25

Keywords: Singular integral equations; Riemann-Hilbert problems; Integral operators; Convolution type

\section{Introduction}

There were rather complete investigations on the method of solution for integral equations of Cauchy type and integral equations of convolution type [1-5]. The solvability of a singular integral equation (SIE) of Wiener-Hopf type with continuous coefficients was considered in [6, 7]. For operators with Cauchy principal value integral and convolution, the conditions of their Noethericity were discussed in [8, 9]. Recently, Li [10-16] studied some classes of SIEs with convolution kernels and gave the Noether theory of solvability and the general solutions in the cases of normal type. It is well known that integral equations of convolution type, mathematically, belong to an interesting subject in the theory of integral equations.

In this paper, we study the solvability and the explicit solutions for several classes of SIEs with Cauchy kernel and convolution kernel, in which include equations with one or two convolution kernels, equation of Wiener-Hopf type, and dual equations. Here, we give the new methods of solution for these equations, and our approach of solving the equations is novel and effective, different from the ones in classical cases. Thus, the results in this

(c) The Author(s) 2019. This article is distributed under the terms of the Creative Commons Attribution 4.0 International License (http://creativecommons.org/licenses/by/4.0/), which permits unrestricted use, distribution, and reproduction in any medium, provided you give appropriate credit to the original author(s) and the source, provide a link to the Creative Commons license, and indicate if changes were made. 
paper generalize ones in Refs. $[1,2,10-12]$, and improve the theory of SIEs and boundary value theory.

Our study of the following equations is motivated by continuous efforts of those studies as well as a sufficiently long list of the above-mentioned materials. Now we consider the following some classes of SIEs of convolution type with Cauchy kernel.

(1) SIEs of dual type

$$
\begin{cases}a_{1} \omega(t)+\frac{b_{1}}{\pi i} \int_{\mathbb{R}} \frac{\omega(\tau)}{\tau-t} d \tau+\frac{1}{\sqrt{2 \pi}} \int_{\mathbb{R}} k_{1}(t-\tau) \omega(\tau) d \tau=g(t), & t \in \mathbb{R}^{+} \\ a_{2} \omega(t)+\frac{b_{2}}{\pi i} \int_{\mathbb{R}} \frac{\omega(\tau)}{\tau-t} d \tau+\frac{1}{\sqrt{2 \pi}} \int_{\mathbb{R}} k_{2}(t-\tau) \omega(\tau) d \tau=g(t), & t \in \mathbb{R}^{-} .\end{cases}
$$

(2) SIE of Wiener-Hopf type

$$
a \omega(t)+\frac{b}{\pi i} \int_{\mathbb{R}} \frac{\omega(\tau)}{\tau-t} d \tau+\frac{1}{\sqrt{2 \pi}} \int_{\mathbb{R}} k(t-\tau) \omega(\tau) d \tau=g(t), \quad t \in \mathbb{R}^{+} .
$$

(3) SIEs with one convolution kernel

$$
a \omega(t)+\frac{b}{\pi i} \int_{\mathbb{R}} \frac{\omega(\tau)}{\tau-t} d \tau+\frac{1}{\sqrt{2 \pi}} \int_{\mathbb{R}} k(t-\tau) \omega(\tau) d \tau=g(t), \quad t \in \mathbb{R} .
$$

(4) SIEs with two convolution kernels

$$
\begin{gathered}
a \omega(t)+\frac{b}{\pi i} \int_{\mathbb{R}} \frac{\omega(\tau)}{\tau-t} d \tau+\frac{1}{\sqrt{2 \pi}} \int_{\mathbb{R}^{+}} k_{1}(t-\tau) \omega(\tau) d \tau \\
+\frac{1}{\sqrt{2 \pi}} \int_{\mathbb{R}^{-}} k_{2}(t-\tau) \omega(\tau) d \tau=g(t), \quad t \in \mathbb{R},
\end{gathered}
$$

where $a, b(b \neq 0), a_{j}, b_{j}$ are constants and $b_{j}$ are not equal to zero simultaneously. For these equations, we will discuss their Noether theory and methods of solution in a different class of function, that is, these equations are transformed by integral transformation into Riemann-Hilbert problems with nodes. Some special kinds of Riemann-Hilbert problems with discontinuous coefficients appear in the course of solution, which are solved in the same time. Actually, the problem to find their solutions is very important in practical applications.

\section{Definitions and lemmas}

The concepts of classes $\{\{0\}\}(((0)),\langle\langle 0\rangle\rangle)$ and $\{0\}((0),\langle 0\rangle)$ are introduced as follows.

Definition 2.1 A function $F(s)$ belongs to $\{\{0\}\}$, if the following two conditions are fulfilled:

(1) $F(s) \in \hat{H}$, that is, it satisfies the Hölder condition on $\mathbb{R} \cup\{\infty\}$ (for the notation $\hat{H}$, cf. [2]).

(2) $F(s) \in L^{2}(\mathbb{R})$.

Definition 2.2 A function $f(t) \in\{0\}$, if its Fourier transform

$$
F(s)=\mathbb{F} f(t)=\frac{1}{\sqrt{2 \pi}} \int_{\mathbb{R}} f(t) e^{i s t} d t, \quad s \in \mathbb{R},
$$

belongs to $\{\{0\}\}$. 
Definition 2.3 Let $F(s)$ be a continuous function on $\mathbb{R}$. If

(1) $F(s) \in \hat{H}$. (2) $F(s)=O\left(|s|^{-\rho}\right), \rho>\frac{1}{2}$, where $|s|$ is sufficiently large.

Then we write $F(s) \in((0))$ or $((0))^{\rho}$.

If $F(s) \in((0))$ or $((0))^{\rho}$, we write $f(t) \in(0)$ or $(0)^{\rho}$.

Definition 2.4 If (1) $F(s) \in \hat{H}$; (2) $F(s) \in H^{\rho}\left(N_{\infty}\right), \rho>\frac{1}{2}$, i.e., it belongs to $H$ in the neighborhood $N_{\infty}$ of $\infty$, and $F(\infty)=0$.

Then we write $F(s) \in\langle\langle 0\rangle\rangle$ or $\langle\langle 0\rangle\rangle^{\rho}$, and $f(t) \in\langle 0\rangle$ or $\langle 0\rangle^{\rho}$.

Let $H_{1}=\left\{F(s) \mid F(s) \in L^{2}(\mathbb{R})\right\}, H_{2}=\left\{F(s) \mid F(s)=O\left(|s|^{-\rho}\right), \rho>\frac{1}{2}\right\}$, and

$$
H_{3}=\left\{F(s) \mid F(s) \in H^{\rho}\left(N_{\infty}\right), \rho>\frac{1}{2}\right\} .
$$

Obviously, $H_{3} \subset H_{2} \subset H_{1}$, hence $\langle\langle 0\rangle\rangle \subset((0)) \subset\{\{0\}\}$, and $\langle 0\rangle \subset(0) \subset\{0\}$.

We denote by $H_{0}$ the class of Hölder continuous functions on any closed interval exterior to $s=0$.

For two functions $k(t)$ and $f(t)$, their convolution is defined by the formula

$$
k * f(t)=\frac{1}{\sqrt{2 \pi}} \int_{\mathbb{R}} k(t-\tau) f(\tau) d \tau, \quad t \in \mathbb{R} .
$$

It is well known that $\mathbb{F}(k * f)=K F$, where $K, F$ are the Fourier transforms of $k, f$, respectively.

Lemma 2.1 is obvious fact and we omit its proof here.

\section{Lemma 2.1}

(1) If $k, f \in\{0\}(\langle 0\rangle)$, then $k * f \in\{0\}(\langle 0\rangle)$.

(2) If $f \in\{0\}$ and $k \in(0)(\langle 0\rangle)$, then $k * f \in(0)(\langle 0\rangle)$.

We also introduce the Cauchy operator T:

$$
T f(t)=\frac{1}{\pi i} \int_{\mathbb{R}} \frac{f(\tau)}{\tau-t} d \tau, \quad t \in \mathbb{R}
$$

It follows from $[15,16]$ that $T$ maps $\{0\}$ and $\langle 0\rangle$ into themselves, respectively, and $T^{2}=I$ (identity).

We define the operators $N$ and $S$ as follows:

$$
N f(t)=f(-t), \quad S f(t)=f(t) \operatorname{sgn} t,
$$

obviously, $N^{2}=S^{2}=I$, and $S N=-N S$.

For the inverse Fourier transform operator $\mathbb{F}^{-1}$ :

$$
\mathbb{F}^{-1} F=\frac{1}{\sqrt{2 \pi}} \int_{\mathbb{R}} F(s) e^{-i s t} d s, \quad t \in \mathbb{R} .
$$

It is evident that

$$
\mathbb{F}^{-1}=N \mathbb{F}=\mathbb{F} N, \quad \mathbb{F}^{2}=N,
$$


and for any $f \in\{0\}$, we have

$$
\mathbb{F} S f=T \mathbb{F} f \text {. }
$$

Lemma 2.2 plays an important role in our paper.

Lemma 2.2 Let $f(t) \in\{0\}, F(s)=\mathbb{F} f(t)$, then $\mathbb{F}[T f(t)]=-S F(s)$, that is,

$$
\mathbb{F}\left[\frac{1}{\pi i} \int_{\mathbb{R}} \frac{f(\tau)}{\tau-t} d \tau\right]=-F(s) \operatorname{sgn} s .
$$

Proof Since

$$
\begin{aligned}
\mathbb{F}[T f(t)] & =\frac{1}{\sqrt{2 \pi}} \int_{\mathbb{R}}\left[\frac{1}{\pi i} \int_{\mathbb{R}} \frac{f(\tau)}{\tau-t} d \tau\right] e^{i s t} d t \\
& =-\frac{1}{\sqrt{2 \pi}} \int_{\mathbb{R}}\left[\frac{1}{\pi i} \int_{\mathbb{R}} \frac{e^{i s t}}{t-\tau} d t\right] f(\tau) d \tau,
\end{aligned}
$$

by the extended residue theorem [1], we have

$$
\frac{1}{\pi i} \int_{\mathbb{R}} \frac{e^{i s t}}{t-\tau} d t= \begin{cases}e^{i s \tau}, & \text { if } s>0, \\ 0, & \text { if } s=0, \\ -e^{i s \tau}, & \text { if } s<0 .\end{cases}
$$

Substituting (2.10) into (2.9), we obtain

$$
\mathbb{F}[T f(t)]=-\operatorname{sgn} s \cdot \frac{1}{\sqrt{2 \pi}} \int_{\mathbb{R}} f(t) e^{i s t} d t=-\operatorname{sgn} s F(s) .
$$

Lemma 2.3 If $\in\{0\}$ and $F(0)=0$, then $T f \in\{0\}$.

Proof Since $f \in\{0\}$, then $F=\mathbb{F} f \in\{\{0\}\}$ and $F \in C(\mathbb{R})$. By $F(0)=0$ and $F(s) \in L^{2}(\mathbb{R})$, we have $F(s) \operatorname{sgn} s \in C(\mathbb{R}) \cup L^{2}(\mathbb{R})$. For any two points $s_{1}, s_{2}$ in the neighborhood of $+\infty$ (or $-\infty)$, that is, there exists a sufficiently large constant $M$ such that $s_{j}>M\left(\right.$ or $\left.s_{j}<-M\right)$. Since

$$
\left|F\left(s_{1}\right) \operatorname{sgn} s_{1}-F\left(s_{2}\right) \operatorname{sgn} s_{2}\right|=\left|F\left(s_{1}\right)-F\left(s_{2}\right)\right|,
$$

we can obtain $F(s) \operatorname{sgn} s \in H^{\rho}$ from $F(s) \in H^{\rho}$, where $\rho$ is the Hölder exponent.

On the other hand, for any $s_{1}, s_{2} \in[-M, M]$, when $s_{1} s_{2}>0,(2.12)$ is fulfilled; when $s_{1} s_{2} \leq$ 0 , since

$$
\begin{aligned}
\left|F\left(s_{1}\right) \operatorname{sgn} s_{1}-F\left(s_{2}\right) \operatorname{sgn} s_{2}\right| & =\left|F\left(s_{1}\right)+F\left(s_{2}\right)\right| \\
& =\left|F\left(s_{1}\right)-F(0)+F\left(s_{2}\right)-F(0)\right| \\
& \leq\left|F\left(s_{1}\right)-F(0)\right|+\left|F\left(s_{2}\right)-F(0)\right| \\
& \leq D_{1}\left|s_{1}\right|^{\rho_{1}}+D_{2}\left|s_{2}\right|^{\rho_{2}} \leq\left(D_{1}+D_{2}\right)\left|s_{1}-s_{2}\right|^{\rho},
\end{aligned}
$$


Li Journal of Inequalities and Applications

(2019) 2019:22

Page 5 of 19

$F(s) \operatorname{sgn} s \in \hat{H}$, where $D_{j}(j=1,2)$ are positive constants, and

$$
\rho= \begin{cases}\max \left\{\rho_{1}, \rho_{2}\right\}, & \text { if }\left|s_{1}-s_{2}\right| \geq 1 \\ \min \left\{\rho_{1}, \rho_{2}\right\}, & \text { if }\left|s_{1}-s_{2}\right|<1\end{cases}
$$

Through the above discussion, we obtain $F(s) \operatorname{sgn} s \in\{\{0\}\}$ and then $T f \in\{0\}$.

In Lemma 2.3, note that $F(0)=0$ is a necessary condition, otherwise the lemma is invalid. Similarly, we can prove that, if $f \in(0)(\langle 0\rangle)$, and $F(0)=0$, then $T f \in(0)(\langle 0\rangle)$.

In order to transform the above-mentioned SUEs into Riemann-Hilbert problems, we give Lemma 2.4 .

Lemma 2.4 Let $\omega(t) \in\{0\}, \Omega(x)=\mathbb{F} \omega(t)$, and $\tilde{\Omega}(z)=\frac{1}{2 \pi i} \int_{\mathbb{R}} \frac{\Omega(s)}{s-z} d s$, then, we have

(1) $\operatorname{Im} z>0, \tilde{\Omega}(z)=\frac{1}{\sqrt{2 \pi}} \int_{\mathbb{R}^{+}} \omega(t) e^{i t z} d t$.

(2) $\operatorname{Im} z<0, \tilde{\Omega}(z)=-\frac{1}{\sqrt{2 \pi}} \int_{\mathbb{R}^{-}} \omega(t) e^{i t z} d t$.

(3) $\operatorname{Im} z=0, \tilde{\Omega}(z)=\frac{1}{\sqrt{2 \pi}} \int_{\mathbb{R}} \omega(t) e^{i t z} \operatorname{sgn} t d t$.

Proof Since $\omega(t) \in\{0\}$, we have

$$
\begin{aligned}
\tilde{\Omega}(z) & =\frac{1}{2 \pi i} \int_{\mathbb{R}} \frac{1}{s-z}\left[\frac{1}{\sqrt{2 \pi}} \int_{\mathbb{R}} \omega(t) e^{i s t} d t\right] d s \\
& =\frac{1}{\sqrt{2 \pi}} \int_{\mathbb{R}^{-}} \omega(t) d t \frac{1}{2 \pi i} \int_{\mathbb{R}} \frac{e^{i s t}}{s-z} d s+\frac{1}{\sqrt{2 \pi}} \int_{\mathbb{R}^{+}} \omega(t) d t \frac{1}{2 \pi i} \int_{\mathbb{R}} \frac{e^{i s t}}{s-z} d s .
\end{aligned}
$$

Let $\Theta=\frac{1}{2 \pi i} \int_{\mathbb{R}} \frac{e^{i s t}}{s-z} d s$. By the generalized residue theorem, when $\operatorname{Im} t>0$, we have

$$
\Theta= \begin{cases}e^{i t z}, & \operatorname{Im} z>0 \\ 0, & \operatorname{Im} z<0\end{cases}
$$

and when $\operatorname{Im} t<0$,

$$
\Theta= \begin{cases}-e^{-i t z}, & \operatorname{Im} z<0 \\ 0, & \operatorname{Im} z>0\end{cases}
$$

Therefore, when $\operatorname{Im} z>0$, we get

$$
\frac{1}{\sqrt{2 \pi}} \int_{\mathbb{R}^{-}} \omega(t) d t \frac{1}{2 \pi i} \int_{\mathbb{R}} \frac{e^{i s t}}{s-z} d s=0
$$

and

$$
\frac{1}{\sqrt{2 \pi}} \int_{\mathbb{R}^{+}} \omega(t) d t \frac{1}{2 \pi i} \int_{\mathbb{R}} \frac{e^{i s t}}{s-z} d s=\frac{1}{\sqrt{2 \pi}} \int_{\mathbb{R}^{+}} \omega(t) e^{i t z} d t
$$

when $\operatorname{Im} z<0$, we have

$$
\frac{1}{\sqrt{2 \pi}} \int_{\mathbb{R}^{+}} \omega(t) d t \frac{1}{2 \pi i} \int_{\mathbb{R}} \frac{e^{i s t}}{s-z} d s=0
$$


and

$$
\frac{1}{\sqrt{2 \pi}} \int_{\mathbb{R}^{-}} \omega(t) d t \frac{1}{2 \pi i} \int_{\mathbb{R}} \frac{e^{i s t}}{s-z} d s=-\frac{1}{\sqrt{2 \pi}} \int_{\mathbb{R}^{-}} \omega(t) e^{i t z} d t ;
$$

when $\operatorname{Im} z=0$, by applying Plemelj formula [12], we find

$$
\tilde{\Omega}^{+}(x)=\frac{1}{2} \Omega(x)+\tilde{\Omega}(x), \quad \tilde{\Omega}^{-}(x)=-\frac{1}{2} \Omega(x)+\tilde{\Omega}(x),
$$

therefore,

$$
\tilde{\Omega}(x)=\frac{1}{2}\left[\tilde{\Omega}^{+}(x)+\tilde{\Omega}^{-}(x)\right]
$$

and

$$
\tilde{\Omega}^{+}(x)=\frac{1}{\sqrt{2 \pi}} \int_{\mathbb{R}^{+}} \omega(t) e^{i t x} d t ; \quad \tilde{\Omega}^{-}(x)=-\frac{1}{\sqrt{2 \pi}} \int_{\mathbb{R}^{-}} \omega(t) e^{i t x} d t .
$$

Then, we have

$$
\begin{aligned}
\tilde{\Omega}(x) & =\frac{1}{2 \pi i} \int_{\mathbb{R}} \frac{\Omega(s)}{s-x} d s=\frac{1}{\sqrt{2 \pi}} \int_{\mathbb{R}^{+}} \omega(t) e^{i t x} d t-\frac{1}{\sqrt{2 \pi}} \int_{\mathbb{R}^{-}} \omega(t) e^{i t x} d t \\
& =\frac{1}{\sqrt{2 \pi}} \int_{\mathbb{R}} \omega(t) e^{i t x} \operatorname{sgn} t d t .
\end{aligned}
$$

Remark 2.1 If $\omega(t) \in L^{1}(\mathbb{R})$, then $\Omega(0)=0$ if and only if $\int_{\mathbb{R}} \omega(t) d t=0$.

Remark 2.2 Note that, for the class (0) or $\langle 0\rangle$, the index $\rho$ is invariant, provided $\frac{1}{2}<\rho<1$.

In Sects. 3-6, we shall study the Noether theory of solvability and methods of solution for some classes of SIEs of convolution type with Cauchy kernel.

\section{Dual equations}

Let us consider the following SIEs of dual type:

$$
\begin{cases}a \omega(t)+\frac{b}{\pi i} \int_{\mathbb{R}} \frac{\omega(\tau)}{\tau-t} d \tau+\frac{1}{\sqrt{2 \pi}} \int_{\mathbb{R}} k_{1}(t-\tau) \omega(\tau) d \tau=g(t), & t \in \mathbb{R}^{+} \\ a \omega(t)+\frac{b}{\pi i} \int_{\mathbb{R}} \frac{\omega(\tau)}{\tau-t} d \tau+\frac{1}{\sqrt{2 \pi}} \int_{\mathbb{R}} k_{2}(t-\tau) \omega(\tau) d \tau=g(t), & t \in \mathbb{R}^{-}\end{cases}
$$

where $a, b$ are constants and $b \neq 0$. The given functions $k_{j}, g \in\{0\}(j=1,2)$, and an unknown function $\omega \in\{0\}$, then their Fourier transforms belong to class $\{\{0\}\}$. In order to solve Eq. (3.1), we rewrite it as

$$
\left\{\begin{array}{l}
a \omega(t)+\frac{b}{\pi i} \int_{\mathbb{R}} \frac{\omega(\tau)}{\tau-t} d \tau+\frac{1}{\sqrt{2 \pi}} \int_{\mathbb{R}} k_{1}(t-\tau) \omega(\tau) d \tau=g(t)-f_{-}(t) ; \\
a \omega(t)+\frac{b}{\pi i} \int_{\mathbb{R}} \frac{\omega(\tau)}{\tau-t} d \tau+\frac{1}{\sqrt{2 \pi}} \int_{\mathbb{R}} k_{2}(t-\tau) \omega(\tau) d \tau=g(t)+f_{+}(t),
\end{array} \quad t \in \mathbb{R},\right.
$$

where $f \in\{0\}$ is an undetermined function.

$$
f_{+}(t)=\left\{\begin{array}{ll}
f(t), & t \geq 0, \\
0, & t<0 ;
\end{array} \quad f_{-}(t)= \begin{cases}0, & t \geq 0 \\
-f(t), & t<0\end{cases}\right.
$$


and $f(t)=f_{+}(t)-f_{-}(t)$.

Taking Fourier transforms in both sides of (3.2), by Lemmas 2.1 and 2.2 we obtain

$$
\left\{\begin{array}{l}
a \Omega(s)-b \operatorname{sgn} s \Omega(s)+K_{1}(s) \Omega(s)=G(s)+F^{-}(s) ; \\
a \Omega(s)-b \operatorname{sgn} s \Omega(s)+K_{2}(s) \Omega(s)=G(s)+F^{+}(s),
\end{array}\right.
$$

where $K_{j}(s)=\mathbb{F} k_{j}(t), \Omega(s)=\mathbb{F} \omega(t), G(s)=\mathbb{F} g(t), F^{ \pm}(s)=\mathbb{F} f_{ \pm}(t), j=1,2$.

By Lemma 2.4, we know that $F^{ \pm}(s)$ are the boundary values of the Cauchy type integral

$$
F(z)=\frac{1}{2 \pi i} \int_{\mathbb{R}} \frac{F(\tau)}{\tau-z} d \tau, \quad \operatorname{Im} z \neq 0,
$$

and it is well known that $F^{ \pm}(s)$ also are the one-sided Fourier transforms of $f(t)$, that is,

$$
F^{+}(s)=\frac{1}{\sqrt{2 \pi}} \int_{\mathbb{R}^{+}} f(t) e^{i t s} d t ; \quad F^{-}(s)=-\frac{1}{\sqrt{2 \pi}} \int_{\mathbb{R}^{-}} f(t) e^{i t s} d t .
$$

It is evident that $F(s)=F^{+}(s)-F^{-}(s)$.

Since $\Omega(s)$ is continuous at $s=0$, thus $\Omega(0)=0$ by (3.4). We assume

$$
K_{j}(s) \neq\left\{\begin{array}{ll}
-(a-b), & 0<s<+\infty, \\
-(a+b), & -\infty<s<0,
\end{array} \quad j=1,2,\right.
$$

which is called the normal case of (3.1). By eliminating $\Omega(s)$ in (3.4) we get

$$
\left[a-b \operatorname{sgn} s+K_{1}(s)\right]\left[G(s)+F^{+}(s)\right]=\left[a-b \operatorname{sgn} s+K_{2}(s)\right]\left[G(s)+F^{-}(s)\right],
$$

so that (3.6) may be written as the following Riemann-Hilbert problems with discontinuous coefficients:

$$
F^{+}(s)=\Phi(s) F^{-}(s)+\Upsilon(s), \quad s \in \mathbb{R}
$$

where

$$
\Phi(s)=\frac{a-b \operatorname{sgn} s+K_{2}(s)}{a-b \operatorname{sgn} s+K_{1}(s)}, \quad \Upsilon(s)=\frac{\left[K_{2}(s)-K_{1}(s)\right] G(s)}{a-b \operatorname{sgn} s+K_{1}(s)} .
$$

We remark that the Riemann boundary value problem (3.7) can be directly solved by the methods in [1] under certain conditions through Fredholm integral equations (see also Muskhelishvili [17]). But in this paper we shall apply Fourier theory to solve (3.7), which may enable us to deal with other equations.

Since $K_{j}(s) \in\{\{0\}\}$, then $K_{j}(\infty)=0(j=1,2)$, so $\Phi(\infty)=1$ and $\Upsilon(\infty)=0$. Thus $s=\infty$ is not a nodal point of (3.7), and its unique nodal point is $s=0$. Note that $F(s)$ is continuous on the whole real axis and $F(\infty)=0$. We take a continuous branch of $\log \Phi(s)$ such that it is continuous at $s=\infty$, e.g., $\log \Phi(\infty)=0$, and denote

$$
\frac{1}{2 \pi i}\{\log \Phi(+0)-\log \Phi(-0)\}=\gamma_{0}=\delta_{0}+i \eta_{0} .
$$


We choose an integer $\kappa$ such that $0 \leq \delta=\delta_{0}-\kappa<1$, and call $\kappa$ the index of (3.7). The homogeneous problem of (3.7) is denoted by

$$
F^{+}(s)=\Phi(s) F^{-}(s), \quad s \in \mathbb{R} .
$$

Without loss of generality, we take the fixed point $z_{0}$ such that $\operatorname{Im} z_{0}>0$, and $\bar{z}_{0}$ denotes the conjugate complex number of $z_{0}$. In order to solve (3.10), we define the following sectionally holomorphic function:

$$
V(z)= \begin{cases}\left(z-\bar{z}_{0}\right)^{-\kappa} e^{\Gamma(z)}, & \operatorname{Im} z>0 \\ \left(z-z_{0}\right)^{-\kappa} e^{\Gamma(z)}, & \operatorname{Im} z<0,\end{cases}
$$

where

$$
\Gamma(z)=\frac{1}{2 \pi i} \int_{\mathbb{R}} \frac{\log \Phi_{0}(t)}{t-z} d t
$$

and

$$
\Phi_{0}(t)=\left(\frac{t-z_{0}}{t-\bar{z}_{0}}\right)^{-\kappa} \Phi(t)
$$

in which we have taken the definite branch of

$$
\log \Phi_{0}(t)=-k \log \frac{t-z_{0}}{t-\bar{z}_{0}}+\log \Phi(t)
$$

provided that we have chosen $\left.\log \frac{t-z_{0}}{t-\bar{z}_{0}}\right|_{t=\infty}=0$, or, which is the same, $\left.\log \frac{t-z_{0}}{t-\bar{z}_{0}}\right|_{t=\operatorname{Re} z_{0}}= \pm i \pi$. From (3.11) and (3.12), we have

$$
V^{+}(s)=\Phi(s) V^{-}(s), \quad s \in \mathbb{R} .
$$

\section{Since}

$$
\frac{F^{+}(s)}{V^{+}(s)}=\frac{F^{-}(s)}{V^{-}(s)},
$$

$D(z)=\frac{F(z)}{V(z)}$ is analytic on $\mathbb{R}^{2}$ and has the order $\kappa-1$ at $\infty$. By the generalized Liouville theorem [14], we can get a general solution of (3.10),

$$
F(z)=V(z) P_{\kappa-1}(z),
$$

and when $\kappa>0, P_{\kappa-1}(z)=e_{0}+e_{1} z+\cdots+e_{\kappa-1} z^{\kappa-1}$ is a polynomial of degree $\kappa-1$; when $\kappa \leq 0, P_{\kappa-1}(z) \equiv 0$, that is, (3.10) only has zero solution.

Next, we consider the solutions and the conditions of solvability for (3.7). To do this, we define $Y(z)$ as follows:

$$
Y(z)= \begin{cases}e^{\Gamma(z)}, & \operatorname{Im} z>0 ; \\ \left(\frac{z-z_{0}}{z-\bar{z}_{0}}\right)^{-\kappa} e^{\Gamma(z),}, & \operatorname{Im} z<0 .\end{cases}
$$


According to the method used in [17-19], (3.7) can be transformed into

$$
\frac{F^{+}(s)}{Y^{+}(s)}=\frac{F^{-}(s)}{Y^{-}(s)}+\frac{\Upsilon(s)}{Y^{+}(s)} .
$$

We again define the following sectionally holomorphic function:

$$
\Psi(z)=\frac{1}{2 \pi i} \int_{\mathbb{R}} \frac{\Upsilon(t)}{Y^{+}(t)(t-z)} d t, \quad z \bar{\in} \mathbb{R}
$$

By applying Plemelj's formula to $\Psi(z)$ in (3.18), we rewrite (3.17) as

$$
\frac{F^{+}(s)}{Y^{+}(s)}-\Psi^{+}(s)=\frac{F^{-}(s)}{Y^{-}(s)}-\Psi^{-}(s) .
$$

It is easily seen that,

$$
D(z)=\frac{F(z)}{Y(z)}-\Psi(z)
$$

is possibly analytic exterior to $z=\bar{z}_{0}$. In order to guarantee that $D(z)$ is bounded at $z=\bar{z}_{0}$, we have that

$$
\left(z-\bar{z}_{0}\right)^{\kappa} D(z)=P_{\kappa-1}(z)
$$

Thus, the general solution of Eq. (3.7) is given by formula

$$
F(z)=Y(z)\left[\Psi(z)+\frac{P_{\kappa-1}(z)}{\left(z-\bar{z}_{0}\right)^{\kappa}}\right], \quad \kappa>0,
$$

and

$$
P_{\kappa-1}(z)=e_{0}+e_{1} z+\cdots+e_{\kappa-1} z^{\kappa-1}
$$

which is a polynomial of degree $\kappa-1$ with arbitrary complex coefficients. If $\kappa \leq 0$, then $P_{\kappa-1}(z) \equiv 0$, in this case, (3.7) has the unique solution

$$
F(z)=Y(z) \Psi(z) .
$$

And when $\kappa \leq-1$, (3.7) is solvable if the conditions

$$
\int_{\mathbb{R}} \frac{\Upsilon(t)}{Y^{+}(t)}\left(z-\bar{z}_{0}\right)^{-j} d t=0, \quad j=1,2, \ldots,-\kappa
$$

are also satisfied.

By taking the boundary values to $Y(z)$ in (3.16), we have

$$
Y^{+}(t)=\sqrt{\Phi_{0}(t)} e^{\Gamma(t)}, \quad Y^{-}(t)=\frac{1}{\sqrt{\Phi_{0}(t)}} e^{\Gamma(t)},
$$


where $\sqrt{\Phi_{0}(t)}=\exp \left\{\frac{1}{2} \log \Phi_{0}(t)\right\}$ has definite value. By applying the Plemelj formula to (3.20), we get

$$
\begin{aligned}
& F^{+}(s)=\frac{1}{2} \Upsilon(s)+Y^{+}(s)\left[\Psi(s)+\frac{Q_{k-1}(s)}{\left(s-\bar{z}_{0}\right)^{k}}\right] \\
& F^{-}(s)=-\frac{1}{2} \frac{\Upsilon(s)}{\Phi(s)}+Y^{-}(s)\left[\Psi(s)+\frac{Q_{k-1}(s)}{\left(s-\bar{z}_{0}\right)^{k}}\right] .
\end{aligned}
$$

Therefore, the general solution of (3.7) may also be written in the following form:

$$
F(s)=\frac{1+\Phi(s)}{2 \Phi(s)} \Upsilon(s)+\left[Y^{+}(s)-Y^{-}(s)\right]\left[\Psi(s)+\frac{Q_{k-1}(s)}{\left(s-\bar{z}_{0}\right)^{k}}\right] .
$$

Since $Y^{ \pm}(s)$ are bounded and nonzero on $\mathbb{R}$, we can prove that $F^{ \pm}(s), F(s) \in L^{2}(\mathbb{R}) \cap H_{0}$. In order to guarantee that $F^{ \pm}(s), F(s) \in\{\{0\}\}$, we need to consider some properties of $F^{ \pm}(s)$, $F(s)$ at $s=0$.

Now we discuss the behaviors of the solution near $s=0$.

(1) Let $s=0$ be an ordinary node. Then, $0<\delta<1, \gamma=\gamma_{0}-\kappa=\delta+i \eta_{0} \neq 0$ and $e^{\gamma \pi i} \neq 1$. It is easy to verify that, in the neighborhood of $s=0$,

$$
Y^{+}(s)=\sqrt{\Phi_{0}(s)} s^{\gamma} e^{\Gamma_{0}(s)}, \quad \Gamma_{0}(s) \in H,
$$

where $\Gamma_{0}(s)=\Gamma(s)-\gamma \ln s$. Moreover, we can prove that,

$$
\begin{aligned}
& \sqrt{\Phi_{0}( \pm 0)}=\exp \frac{1}{2}[ \pm \kappa \pi i+\log \Phi( \pm 0)] ; \\
& \sqrt{\Phi_{0}(-0)}=e^{\gamma \pi i} \sqrt{\Phi_{0}(+0)} .
\end{aligned}
$$

Similar to the discussion in $[12,20,21]$, when $s>0$, we have

$$
\Psi(s)=\frac{i \csc \gamma \pi}{2 s^{\gamma} e^{\Gamma_{0}(s)}}\left[e^{-\gamma \pi i} \frac{\Upsilon(-0)}{\sqrt{\Phi_{0}(-0)}}-\cos \gamma \pi \frac{\Upsilon(+0)}{\sqrt{\Phi_{0}(+0)}}\right]+A(s),
$$

where $A(s)=\frac{\Psi^{*}(s)}{|s|^{\delta^{\prime}}}\left(0<\delta^{\prime}<\delta\right)$ and $\Psi^{*}(s) \in H$. We apply (3.26) to simplify (3.24) and obtain

$$
F^{+}(+0)=\frac{i \csc \gamma \pi}{2 e^{2 \gamma \pi i}}\left[\Upsilon(-0)-e^{3 \gamma \pi i} \Upsilon(+0)\right] .
$$

When $s<0$, we have

$$
\Psi(s)=\frac{i \csc \gamma \pi}{2 s^{\gamma} e^{\Gamma_{0}(s)}}\left[\cos \gamma \pi \frac{\Upsilon(-0)}{\sqrt{\Phi_{0}(-0)}}-e^{\gamma \pi i} \frac{\Upsilon(+0)}{\sqrt{\Phi_{0}(+0)}}\right]+A(s),
$$

and then

$$
F^{+}(-0)=\frac{i \csc \gamma \pi}{2 e^{\gamma \pi i}}\left[\Upsilon(-0)-e^{3 \gamma \pi i} \Upsilon(+0)\right] .
$$

By comparing (3.28) with (3.30), we know that $F(s)$ is continuous at $s=0$ if and only if

$$
\Upsilon(-0)=e^{3 \gamma \pi i} \Upsilon(+0)
$$


Therefore, we obtain

$$
F^{+}(0)=F^{-}(0)=0
$$

and

$$
F(0)=0 \text {. }
$$

By (3.4), we also have

$$
\Omega(0)=0
$$

and

$$
G(0)=0 \text {. }
$$

Conversely, if (3.35) is fulfilled, then (3.32) and (3.33) are valid, and $F^{ \pm}(s), F(s) \in H$ in the neighborhood of $s=0$. Therefore, we have $F^{ \pm}(s), F(s) \in\{\{0\}\}$. In conclusion, it is necessary that $G(0)=0$.

(2) Let $s=0$ be a special node. In this case, $\delta=0$ and $\gamma=i \eta_{0}$.

If $\eta_{0}=0$, that is, $\gamma=0$, then $\Phi(s)$ must be continuous at $s=0$. It follows from $b \neq 0$ that $K_{1}(0)=K_{2}(0)=0$, so $E(0)=1, \Upsilon(0)=0$, and $F(0)=0$. Thus, $F(s)$ is continuous at $s=0$, and $s=0$ is not a nodal point at all. There is no boundary value problem in this case.

If $\eta_{0} \neq 0$, then (3.27) and (3.29) remain valid. We can prove that $A(s) \in H_{0}$ and $A( \pm 0)$ exist, thus we have

$$
F^{+}(+0)=\frac{i \csc \gamma \pi}{2 e^{2 \gamma \pi i}}\left[\Upsilon(-0)-e^{3 \gamma \pi i} \Upsilon(+0)\right]+\lim _{s \rightarrow+0} \sqrt{\Phi_{0}(+0)} e^{\Gamma_{0}(0)} s^{i \eta_{0}}\left[A(s)+B_{0}\right]
$$

where $B_{0}=\left(\bar{z}_{0}\right)^{\kappa} e_{0}$ when $\kappa>0$; and $B_{0}=0$ when $\kappa \leq 0$. And a similar formula for $F^{+}(-0)$ can be obtained, that is,

$$
F^{+}(-0)=\frac{i \csc \gamma \pi}{2 e^{2 \gamma \pi i}}\left[\Upsilon(-0)-e^{3 \gamma \pi i} \Upsilon(+0)\right]+\lim _{s \rightarrow-0} \sqrt{\Phi_{0}(-0)} e^{\Gamma_{0}(0)} s^{i \eta_{0}}\left[A(s)+B_{0}\right] .
$$

In order that $F^{+}( \pm 0)$ exist, we should have

$$
A( \pm 0)= \begin{cases}\left(\bar{z}_{0}\right)^{\kappa} e_{0}, & \kappa>0 \\ 0, & \kappa \leq 0 .\end{cases}
$$

By (3.28), (3.30) and (3.31), we get (3.35) again. Once (3.35) is fulfilled, then $\Upsilon(0)=0$ and $\Psi(s) \in H$ near $s=0$. Since we require $F^{+}(s)$ is continuous at $s=0$, thus, when $\kappa>0$, the constant term $e_{0}$ of $P_{\kappa-1}(z)$ should take the value

$$
e_{0}=\frac{(-1)^{\kappa-1}\left(\bar{z}_{0}\right)^{\kappa}}{2 \pi i} \int_{\mathbb{R}} \frac{\Upsilon(t)}{Y^{+}(t) t} d t
$$

and when $\kappa \leq 0$, the following condition of solvability must be satisfied:

$$
\int_{\mathbb{R}} \frac{\Upsilon(t)}{Y^{+}(t) t} d t=0
$$


Once (3.38) and (3.39) are fulfilled, we can readily verify $F^{ \pm}(0)=F(0)=0$. In all of the above cases, it is easy to see that $F^{ \pm}(s), F(s) \in\{\{0\}\}$.

Moreover, we have the following results.

Theorem 3.1 Assume that (3.5) and (3.35) are fulfilled.

(1) Let $s=0$ be an ordinary node, when $\kappa \geq 0$, (3.1) always has a solution; when $\kappa<0$, provided that (3.22) are fulfilled, (3.1) has the unique solution (3.21).

(2) Let $s=0$ be a special node, when $K_{1}(0)=K_{2}(0)$, the results obtained in (1) remain true; when $K_{1}(0) \neq K_{2}(0)$, if $\kappa>0$ and (3.38) is fulfilled, (3.1) has a solution; if $\kappa \leq 0$, when (3.22) and (3.39) are fulfilled, (3.1) is solvable.

Under the above suppositions, Eq. (3.1) is solvable in class $\{0\}$ and has the solution $\omega(t)=$ $\mathbb{F}^{-1} \Omega(s)$, where $\Omega(s)$ is given by (3.6). Obviously, $F(s) \in\{\{0\}\}$ and so $\omega(t) \in\{0\}$.

Remark 3.1 In Eq. (3.1), if $k_{j} \in\{0\}(j=1,2), g \in(0)$, then $\omega \in(0)$; if $k_{j}, g \in\langle 0\rangle(j=1,2)$, then $\omega \in\langle 0\rangle$. Similarly, we can also obtain that, if $k_{j}, g \in(0)^{\rho}(j=1,2)$, then $\omega \in(0)^{\rho}$, and if $k_{j}, g \in\langle 0\rangle^{\rho}(j=1,2)$, then $\omega \in\langle 0\rangle^{\rho}$, provided $0<\rho<1$.

\section{Equation of Wiener-Hopf type}

In this section, we discuss method of solution for the following SIE of Wiener-Hopf type:

$$
a \omega(t)+\frac{b}{\pi i} \int_{\mathbb{R}} \frac{\omega(\tau)}{\tau-t} d \tau+\frac{1}{\sqrt{2 \pi}} \int_{\mathbb{R}} k(t-\tau) \omega(\tau) d \tau=g(t), \quad t \in \mathbb{R}^{+},
$$

where $a, b$ are as the above, $k(t), g(t) \in(0)^{\rho}\left(\frac{1}{2}<\rho<1\right)$ and $\omega(t)$ is required to be in $\{0\}$.

Extending $t$ in (4.1) to $t \in \mathbb{R}$, the right-hand side of (4.1) is augmented with an unknown function $-\omega_{-}(t)$. Rewrite (4.1) as

$$
a \omega_{+}(t)+\frac{b}{\pi i} \int_{\mathbb{R}} \frac{\omega_{+}(\tau)}{\tau-t} d \tau+\frac{1}{\sqrt{2 \pi}} \int_{\mathbb{R}} k(t-\tau) \omega_{+}(\tau) d \tau=g(t)-\omega_{-}(t), \quad t \in \mathbb{R}
$$

Taking Fourier transformations on both sides of (4.2), we get by Lemma 2.2

$$
a \Omega^{+}(s)-b \operatorname{sgn} s \Omega^{+}(s)+K(s) \Omega^{+}(s)=G(s)+\Omega^{-}(s),
$$

that is,

$$
\Omega^{+}(s)=\Phi(s) \Omega^{-}(s)+W(s),
$$

where

$$
\Phi(s)=\frac{1}{a-b \operatorname{sgn} s+K(s)}, \quad W(s)=\frac{G(s)}{a-b \operatorname{sgn} s+K(s)} .
$$

Note that $\Omega^{ \pm}(s), G(s)$ belong to class $\{\{0\}\}$ on $\mathbb{R}$. Here we only consider the case of normal type, that is,

$$
K(s) \neq \begin{cases}-(a-b), & 0<s<+\infty, \\ -(a+b), & -\infty<s<0 .\end{cases}
$$


Denote

$$
\begin{aligned}
& \frac{1}{2 \pi i}\{\log \Phi(+\infty)-\log \Phi(-\infty)\} \\
& \quad=\frac{1}{2 \pi i}\{\log (a-b)-\log (a+b)\} \\
& \quad=\delta_{\infty}+i \eta_{\infty}=\gamma_{\infty},
\end{aligned}
$$

where $\log \Phi(s)$ is taken to be continuous branch for $s>0$ and $s<0$ respectively such that it is continuous at $s=\infty$, and $0 \leq \delta_{\infty}<1$. Since $b \neq 0$, thus $\gamma_{\infty} \neq 0$. We again denote

$$
\frac{1}{2 \pi i}\{\log \Phi(+0)-\log \Phi(-0)\}=\delta_{0}+i \eta_{0}=\gamma_{0} .
$$

Then we choose an integer $\kappa$, the index of (4.4), such that $0 \leq \delta=\delta_{0}-\kappa<1$. Denote $\gamma=$ $\gamma_{0}-\kappa=\delta+i \eta_{0}$. Note that $\Omega^{+}(\infty)=0$, then we also have $\Omega^{-}(\infty)=\Omega(\infty)=0$. Therefore, it is necessary that $\Omega(\infty)=0$. Since $b \neq 0$, we get $\gamma \neq 0$. It is seen from the above discussion that both $s=0$ and $s=\infty$ are nodes of (4.4).

Similar to the discussion in $[17,22,23]$ and Sect. 3 , we can obtain the general solution of (4.4)

$$
\Omega(z)=Y(z)\left[M(z)+\frac{P_{\kappa-1}(z)}{\left(z-\bar{z}_{0}\right)^{\kappa}}\right]
$$

where

$$
M(z)=\frac{1}{2 \pi i} \int_{\mathbb{R}} \frac{z-\bar{z}_{0}}{t-\bar{z}_{0}} \frac{W(t)}{Y^{+}(t)(t-z)} d t, \quad z \bar{\in} \mathbb{R}
$$

when $\kappa>0, P_{\kappa-1}(z)=e_{0}+e_{1} z+\cdots+e_{\kappa-1} z^{\kappa-1}$ is an arbitrary polynomial of degree $\kappa-1$, and $Y(z), z_{0}$ are still given by (3.16); when $\kappa \leq 0, P_{\kappa-1}(z) \equiv 0$, and the following conditions of solvability must be fulfilled:

$$
\int_{\mathbb{R}} \frac{W(t)}{Y^{+}(t)\left(t-\bar{z}_{0}\right)^{j}} d t=0, \quad j=1,2, \ldots,-\kappa+1
$$

Note that, in (4.10), since $k, g \in(0)$, then $W(\infty)=0$, by [12] we get

$$
M(s)=\frac{M_{*}(s)}{|s|^{\delta^{\prime}}}, \quad 0<\delta^{\prime}<\delta,
$$

where $M_{*}(s) \in H$. Therefore, a factor $\frac{z-\bar{z}_{0}}{t-\bar{z}_{0}}$ should be multiplied in the integrand of (4.10). And we also have $Y^{-}(s)=\frac{\xi(s)}{s^{\gamma} \infty}$, here $\xi(s) \in H_{2}$.

Taking the boundary values for $\Omega(z)$ in (4.9), we have

$$
\Omega^{+}(s)=\frac{1}{2} W(s)+Y^{+}(s)\left[M(s)+\frac{P_{\kappa-1}(s)}{\left(s-\bar{z}_{0}\right)^{\kappa}}\right]
$$

and

$$
\Omega^{-}(s)=-\frac{1}{2} \frac{W(s)}{E(s)}+Y^{-}(s)\left[M(s)+\frac{P_{k-1}(s)}{\left(s-\bar{z}_{0}\right)^{k}}\right] .
$$


In the following, we consider some properties of the solutions at nodes. First, we consider the situation near $s=\infty$.

(1) Let $s=\infty$ be an ordinary node, we have the following two cases.

(a) When $0 \leq \delta_{\infty} \leq \frac{1}{2}$, we easily find that $W(s)[Y(s)]^{-1} \in H_{2}$ since $W(s) \in H_{2}$.

In order to guarantee that $\Omega(s) \in\{\{0\}\}$, when $\kappa \geq 0$, the constant term $e_{0}$ of $P_{\kappa-1}(z)$ should take the value

$$
e_{0}=-\frac{1}{2 \pi i} \int_{\mathbb{R}} \frac{W(t)}{Y^{+}(t)\left(t-\bar{z}_{0}\right)} d t
$$

and when $\kappa<0$, we have the following conditions of solvability:

$$
\int_{\mathbb{R}} \frac{W(t)}{Y^{+}(t) t\left(t-\bar{z}_{0}\right)^{j}} d t=0, \quad j=1,2, \ldots,-\kappa .
$$

(b) When $\frac{1}{2}<\delta_{\infty}<1$, if $\rho \leq \delta_{\infty}$, by [20] we have

$$
Y(s) M(s)=O\left(|s|^{-\delta \infty+\varepsilon}\right)
$$

where $\varepsilon>0$ is arbitrarily small such that $\delta_{\infty}-\varepsilon>\frac{1}{2}$; if $\rho>\delta_{\infty}$, then $M(s)$ is bounded and so

$$
Y(s) M(s)=O\left(|s|^{-\delta \infty}\right)
$$

near $s=\infty$. Thus we also obtain $\Omega^{ \pm}(s) \in H_{2}$ and so $\Omega(s) \in H_{2}$. In conclusion, in any case, we have $F(s)=O\left(|s|^{-\mu}\right)$, where $\mu=\min \left\{\rho, \delta_{\infty}-\varepsilon\right\}$, obviously, $\mu>\frac{1}{2}$.

(2) Let $s=\infty$ be a special node, then $\delta_{\infty}=0$ and $\gamma_{\infty}=i \eta_{\infty} \neq 0$. In this case, the discussion is the same as that in (1), and we can obtain $\Omega(s) \in H$ and $\Omega(s) \in H_{2}$.

Next, we consider the behavior of the solution near $s=0$.

Since $\Omega(s)$ is continuous at $s=0$, similar to the discussion in Sect. 3, we may get $W(+0)=$ $e^{-3 \gamma \pi i} W(-0)$. From (4.5), we have $W( \pm 0)=G(0) \Phi( \pm 0)$. Therefore, we can obtain

$$
G(0)\left[\Phi(-0)-e^{3 \gamma \pi i} \Phi(+0)\right]=0 .
$$

Note that $\Phi(+0)=e^{2 \gamma_{0} \pi i} \Phi(-0)$ and $\gamma=\gamma_{0}-\kappa \neq 0$, so

$$
\Phi(-0)-e^{3 \gamma \pi i} \Phi(+0)=\Phi(-0)\left(1-e^{5 \gamma_{0} \pi i}\right) \neq 0
$$

and by (4.16) and (4.17), we get $G(0)=0$ again.

Moreover, in the case $\delta_{\infty}>\frac{1}{2}$, when $\kappa \geq 0$, for the constant term $e_{0}$ of $P_{\kappa-1}(s)$ must be taken the value

$$
e_{0}=\frac{(-1)^{\kappa}\left(\bar{z}_{0}\right)^{\kappa+1}}{2 \pi i} \int_{\mathbb{R}} \frac{W(t)}{Y^{+}(t)\left(t-\bar{z}_{0}\right) t} d t
$$

when $\kappa \leq-1$, there is the following condition of solvability:

$$
\int_{\mathbb{R}} \frac{W(t)}{Y^{+}(t)\left(t-\bar{z}_{0}\right) t} d t=0
$$


It is seen from the above discussions that $\Omega(0)=0, \Omega(s) \in\{\{0\}\}$. In fact, when $\delta_{\infty}>\frac{1}{2}$, we can get $\Omega(s) \in((0))$ and hence $\omega(t) \in(0)$.

Now we can formulate the main results about the solutions of Eq. (4.1) in following form.

Theorem 4.1 Under condition (4.6), the necessary condition of the existence of solution to Eq. (4.1) is $G(0)=0$ in class $\{0\}$.

(1) Let $s=0$ be a node, if $\frac{1}{2}<\delta_{\infty}<1$, when $\kappa \geq 0$, (4.1) always has a solution, and the constant term of $P_{\kappa-1}(s)$ takes value as (4.18); and when $\kappa<0$, (4.11) and (4.19) should be supplemented, then Eq. (4.1) has a solution. If $0 \leq \delta_{\infty} \leq \frac{1}{2}$, when $\kappa \geq 0$, the constant term of $P_{\kappa-1}(s)$ should be taken as (3.34); when $\kappa<0$, the conditions of solvability (3.22) and (3.35) are fulfilled.

(2) Let $s=\infty$ be a node, if $\frac{1}{2}<\delta_{\infty}<1$, when $\kappa \geq 0$, (4.1) has a solution; when $\kappa<0$, provided that (4.11) are satisfied, (4.1) is solvable. If $0 \leq \delta_{\infty} \leq \frac{1}{2}$, when $\kappa \geq 0$, (4.1) has the solution; when $\kappa<0$, the conditions of solvability (4.15) must be augmented.

Assume that the above (1) and (2) are fulfilled, then (4.1) has the following general solution:

$$
\omega^{+}(t)=\mathbb{F}^{-1} \Omega^{+}(s)
$$

where $\Omega^{+}(s)$ is given by (4.12).

Remark 4.1 We should note that $M(z)$ cannot be separated as, in general,

$$
M(z)=\frac{1}{2 \pi i} \int_{\mathbb{R}} \frac{W(t)}{Y^{+}(t)(t-z)} d t-\frac{1}{2 \pi i} \int_{\mathbb{R}} \frac{W(t)}{Y^{+}(t)\left(t-\bar{z}_{0}\right)} d t
$$

because two integrals in (4.20) may be divergent. But, in case (1) of Theorem 4.1, when $\delta_{\infty} \leq \frac{1}{2},(4.20)$ becomes valid, and the integrals in (4.20) are convergent now. In this case, we also have $Y(s) \Psi(s) \in H_{2}$.

Remark 4.2 In Eq. (4.1), if $a \neq 0$ is real, $b$ is purely imaginary, and $k_{1}(t), k_{2}(t)$ are real functions, then (4.1) is a real equation. In this case, $s=0$ is an ordinary node of (4.1). It is also easily seen that the characteristic feature is for $\delta_{\infty}>\frac{1}{2}$ or $\delta_{\infty} \leq \frac{1}{2}$. By (4.7), when $\delta_{\infty}>\frac{1}{2}$, we have

$$
\pi<\arg (a+b)-\arg (a-b)<2 \pi
$$

then, $a+b$ lies in the quadrant 2 or 4 ; when $\delta_{\infty}<\frac{1}{2}$,

$$
0<\arg (a+b)-\arg (a-b)<\pi
$$

and $a+b$ lies in the quadrant 1 or 3 . 


\section{Equations with two convolution kernels}

The above method is applicable to solving the SIEs with two convolution kernels

$$
\begin{gathered}
a \omega(t)+\frac{b}{\pi i} \int_{\mathbb{R}} \frac{\omega(\tau)}{\tau-t} d \tau+\frac{1}{\sqrt{2 \pi}} \int_{\mathbb{R}^{+}} k_{1}(t-\tau) \omega(\tau) d \tau \\
+\frac{1}{\sqrt{2 \pi}} \int_{\mathbb{R}^{-}} k_{2}(t-\tau) \omega(\tau) d \tau=g(t), \quad t \in \mathbb{R},
\end{gathered}
$$

where $a, b$ are as the above, $k_{1}, k_{2}, g \in\langle 0\rangle$ and the unknown function $\omega \in\{0\}$.

Taking the Fourier transform on both sides of (5.1), we obtain

$$
(a-b \operatorname{sgn} s) \Omega(s)+K_{1}(s) \Omega^{+}(s)-K_{2}(s) \Omega^{-}(s)=G(s),
$$

where $\Omega^{ \pm}(s)$ are respectively the Fourier transforms of $\omega_{ \pm}(t)$. In fact, $\Omega^{ \pm}(s)$ also are the boundary values of the holomorphic function

$$
\Omega(z)=\frac{1}{\sqrt{2 \pi}} \int_{\mathbb{R}} \omega(t) e^{i t z} d t
$$

in the upper and the lower half planes, respectively.

In order to guarantee that $\Omega^{ \pm}(s), \Omega(s)$ are continues at $s=0$, it is necessary that $\Omega(0)=0$, that is, $\Omega^{+}(0)=\Omega^{-}(0)$. Restricted to the case of normal type, i.e., $K(s)$ satisfying (4.6). From (5.2), we get the following Riemann-Hilbert problem:

$$
\Omega^{+}(s)=\Phi(s) \Omega^{-}(s)+W(s), \quad s \in \mathbb{R}
$$

in which we have put

$$
\Phi(s)=\frac{a-b \operatorname{sgn} s+K_{2}(s)}{a-b \operatorname{sgn} s+K_{1}(s)}, \quad W(s)=\frac{G(s)}{a-b \operatorname{sgn} s+K_{1}(s)} .
$$

Similarly, we easily verify that $s=\infty$ is not a nodal point of (5.4), and $s=0$ is its unique nodal point. The solution of (5.4) be discussed by using the same method as shown in Sect. 3. The remaining discussions will be omitted also.

\section{Equations with one convolution kernel}

Finally, we solve SIEs with one convolution kernel

$$
a \omega(t)+\frac{b}{\pi i} \int_{\mathbb{R}} \frac{\omega(\tau)}{\tau-t} d \tau+\frac{1}{\sqrt{2 \pi}} \int_{\mathbb{R}} k(t-\tau) \omega(\tau) d \tau=g(t), \quad t \in \mathbb{R},
$$

where $a, b$ are as the above, $k, g \in\{0\}$ and the unknown function $\omega \in\{0\}$. Taking Fourier transforms on both sides of (6.1), we get by Lemma 2.2

$$
a \Omega(s)-b \operatorname{sgn} s \Omega(s)+K(s) \Omega(s)=G(s) .
$$

Note that (6.2) is not a Riemann-Hilbert problem. Without loss of generality, we only discuss the function $a-b \operatorname{sgn} s+K(s) \neq 0(s \in \mathbb{R})$. From (6.2) we get

$$
\Omega(s)=\frac{G(s)}{a-b \operatorname{sgn} s+K(s)} .
$$


Since $G(s)$ is continuous at $s=0$ and

$$
G(0)=0 \text {, }
$$

thus $G(s) \in\{\{0\}\}$. Note that $a-b \operatorname{sgn} s+K(s)$ is bounded and non-zero on $\mathbb{R}$, we get $\Omega(s) \in$ $\{\{0\}\}$ and hence $\omega(t)=\mathbb{F}^{-1} \Omega(s)$ is the solution of (6.1) in $\{0\}$.

Thus, we obtain the following conclusions.

Theorem 6.1 If $k(t), g(t) \in\{0\}$, in the case of normal type, that is, (4.6) is valid, then (6.1) is solvable if and only if (6.4) is satisfied. Assume that this is fulfilled, (6.1) has a solution

$$
\omega(t)=\mathbb{F}^{-1} \Omega(s)
$$

in $\{0\}$, where $\Omega(s)$ is given by (6.3).

After simplification, $\omega(t)$ may be written as

$$
\omega(t)=q(t)-p * q(t)
$$

where $p(t)=\mathbb{F}^{-1} P(s), q(t)=\mathbb{F}^{-1} Q(s)$, in which

$$
P(s)=\frac{K(s)}{a-b \operatorname{sgn} s+K(s)}, \quad Q(s)=\frac{G(s)}{a-b \operatorname{sgn} s} .
$$

Noting that, although $P(s)$ is discontinuous at $s=0$, it would not influence the property $p * q \in\{0\}$ since $G(0)=0$.

\section{Comments}

Here, we shall discuss the equations of dual type in the more general case

$$
\begin{cases}a_{1} \omega(t)+b_{1} T \omega(t)+k_{1} * \omega(t)=g(t), & t \in \mathbb{R}^{+} \\ a_{2} \omega(t)+b_{2} T \omega(t)+k_{2} * \omega(t)=g(t), & t \in \mathbb{R}^{-}\end{cases}
$$

where $a_{j}, b_{j}$ are constants and all the functions appeared belong to $\{0\}$. Assume $b_{1}, b_{2}$ are not equal to zero simultaneously. Actually, (3.1) is a special case of (7.1). In order to solve Eq. (7.1), we rewrite (7.1) as

$$
\left\{\begin{array}{l}
a_{1} \omega(t)+b_{1} T \omega(t)+k_{1} * \omega(t)=g(t)-f_{-}(t) ; \\
a_{2} \omega(t)+b_{2} T \omega(t)+k_{2} * \omega(t)=g(t)+f_{+}(t),
\end{array} \quad t \in \mathbb{R},\right.
$$

where $f_{ \pm}(t)$ are as the above. Taking Fourier transforms in both sides of (7.2), we obtain

$$
\left\{\begin{array}{l}
{\left[a_{1}-b_{1} \operatorname{sgn} s+K_{1}(s)\right] \Omega(s)=G(s)+F^{-}(s)} \\
{\left[a_{2}-b_{2} \operatorname{sgn} s+K_{2}(s)\right] \Omega(s)=G(s)+F^{+}(s) .}
\end{array}\right.
$$


In the case of normal type, that is,

$$
K_{j}(s) \neq \begin{cases}-\left(a_{j}-b_{j}\right), & 0<s<+\infty, \\ -\left(a_{j}+b_{j}\right), & -\infty<s<0\end{cases}
$$

for any $j=1,2$. By eliminating $\Omega(s)$ in (7.3), it gives rise to

$$
F^{+}(s)=\Phi(s) F^{-}(s)+\Upsilon(s), \quad s \in \mathbb{R}
$$

where

$$
\Phi(s)=\frac{a_{2}-b_{2} \operatorname{sgn} s+K_{2}(s)}{a_{1}-b_{1} \operatorname{sgn} s+K_{1}(s)}
$$

and

$$
\Upsilon(s)=\frac{\left[a_{2}-a_{1}-\left(b_{2}-b_{1}\right) \operatorname{sgn} s+K_{2}(s)-K_{1}(s)\right] G(s)}{a_{1}-b_{1} \operatorname{sgn} s+K_{1}(s)} .
$$

Equation (7.5) is a Riemann-Hilbert problem with discontinuous coefficients and nodes $s=0, \infty$, and its method of solution may be made fully analogous to those in Sect. 4. In order that $\Omega(s)$ is continuous at $s=0$, it is necessary that $F(s)$ is continuous at $s=0$ and $F^{ \pm}(0)=-G(0)$. Since $F^{+}(s)$ is continuous at $s=0$, we should again get $G(0)=0$. Hence all the results as stated in Theorem 4.1 remain true and $\omega(t)=\mathbb{F}^{-1} \Omega(s)$, in which $\Omega(s)$ is given by (7.3). The only difference lies in that $\gamma_{\infty}$ and $\gamma$ may be zero, for instance, when $a_{1}=a_{2}$, $b_{1}=b_{2}$, we have $\gamma_{\infty}=0$, then this case may be transformed to that in Sect. 3 . Here, we will not elaborate on the solving method of (7.5).

\section{Conclusions}

In this paper, we study some classes of SIEs with convolution kernels and Cauchy kernels in the different classes of functions. By Fourier transform, these equations are transformed into Riemann-Hilbert problems with discontinuous coefficients. The general solutions denoted by integrals and the solvable conditions are obtained for the equations. Here, our method is different from the classical ones, and it is novel and effective. Thus, this paper generalizes the theory of the classical Riemann-Hilbert problems and SIEs. Meanwhile, we remark that the methods of this paper may be used to solving the above equations in the non-normal case. Indeed, it is possible to study the above-mentioned equation in Clifford analysis, which is similar to that in [24-28]. Further discussion is omitted here.

\section{Acknowledgements}

The author is very grateful to the anonymous referees for their valuable suggestions and comments, which helped to improve the quality of the paper. This research is supported by the Science and Technology Plan Project of Qufu Normal University (xkj 201606).

\section{Funding}

This work was supported by the Science and Technology Plan Project of Qufu Normal University (xkj 201606).

Availability of data and materials

Not applicable. 
Authors' contributions

This entire work has been completed by the author. Analytical solutions were determined by him. The author read and approved the final manuscript.

\section{Publisher's Note}

Springer Nature remains neutral with regard to jurisdictional claims in published maps and institutional affiliations.

Received: 29 June 2018 Accepted: 16 January 2019 Published online: 25 January 2019

\section{References}

1. Lu, J.K.: Boundary Value Problems for Analytic Functions. World Scientific, Singapore (2004)

2. Litvinchuk, G.S.: Solvability Theory of Boundary Value Problems and Singular Integral Equations with Shift. Kluwer Academic Publisers, London (2004)

3. Chuan, L.H., Mau, N.V., Tuan, N.M.: On a class of singular integral equations with the linear fractional Carleman shift and the degenerate kernel. Complex Var. Elliptic Equ. 53(2), 117-137 (2008)

4. Gahov, F.D., Cerskili, J.I.: Equations of convolution type. Moscow (1978)

5. De-Bonis, M.C., Laurita, C.: Numerical solution of systems of Cauchy singular integral equations with constant coefficients. Appl. Math. Comput. 219, 1391-1410 (2012)

6. Begehr, H., Vaitekhovich, T.: Harmonic boundary value problems in half disc and half ring. Funct. Approx. 40(2), 251-282 (2009)

7. Nakazi, T., Yamamoto, T.: Normal singular integral operators with Cauchy kernel. Integral Equ. Oper. Theory 78, 233-248 (2014)

8. Praha, E.K., Valencia, V.M.: Solving singular convolution equations using inverse Fast Fourier Transform. Appl. Math. 57(5), 543-550 (2012)

9. Wójcik, P., Sheshko, M.A., Sheshko, S.M.: Application of Faber polynomials to the approximate solution of singular integral equations with the Cauchy kernel. Differ. Equ. 49(2), 198-209 (2013)

10. Li, P.R., Ren, G.B.: Solvability of singular integro-differential equations via Riemann-Hilbert problem. J. Differ. Equ. 265 , 5455-5471 (2018)

11. Li, P.R., Ren, G.B.: Some classes of equations of discrete type with harmonic singular operator and convolution. Appl. Math. Comput. 284, 185-194 (2016)

12. Li, P.R.: Generalized convolution-type singular integral equations. Appl. Math. Comput. 311, 314-323 (2017)

13. Li, P.R.: Singular integral equations of convolution type with Hilbert kernel and a discrete jump problem. Adv. Differ. Equ. 2017, 360 (2017)

14. Li, P.R.: Some classes of singular integral equations of convolution type in the class of exponentially increasing functions. J. Inequal. Appl. 2017, 307 (2017)

15. Li, P.R.: Two classes of linear equations of discrete convolution type with harmonic singular operators. Complex Var. Elliptic Equ. 61(1), 67-75 (2016)

16. Li, P.R.: Singular integral equations of convolution type with Cauchy kernel in the class of exponentially increasing functions. Appl. Math. Comput. 344-345, 116-127 (2019)

17. Musknelishvilli, N.I: Singular Integral Equations. Nauka, Moscow (2002)

18. Du, H., Shen, J.H.: Reproducing kernel method of solving singular integral equation with cosecant kernel. J. Math. Anal. Appl. 348(1), 308-314 (2008)

19. Hörmander, L.: The Analysis of Linear Partial Differential Operators. I. Springer, Berlin (2003). Reprint of the second (1990) edition

20. Li, P.R.: One class of generalized boundary value problem for analytic functions. Bound. Value Probl. 2015,40 (2015)

21. Gomez, C., Prado, H., Trofimchuk, S.: Separation dichotomy and wavefronts for a nonlinear convolution equation. J. Math. Anal. Appl. 420,1-19 (2014)

22. Li, P.R.: Singular integral equations of convolution type with cosecant kernels and periodic coefficients. Math. Probl. Eng. 2017, Article ID 6148393 (2017)

23. Blocki, Z:: Suita conjecture and Ohsawa-Takegoshi extension theorem. Invent. Math. 193, 149-158 (2013)

24. Gong, Y.F., Leong, L.T., Qiao, T.: Two integral operators in Clifford analysis. J. Math. Anal. Appl. 354, 435-444 (2009)

25. Tuan, N.M., Thu-Huyen, N.T.: The solvability and explicit solutions of two integral equations via generalized convolutions. J. Math. Anal. Appl. 369, 712-718 (2010)

26. Li, P.R.: Linear BVPs and SIEs for generalized regular functions in Clifford analysis. J. Funct. Spaces 2018, Article ID 6967149 (2018)

27. Abreu-Blaya, R., Bory-Reyes, J., Brackx, F., De-Schepper, H., Sommen, F.: Cauchy integral formulae in Hermitian quaternionic Clifford analysis. Complex Anal. Oper. Theory 6, 971-985 (2012)

28. Colliander, J., Keel, M., Staffilani, G. et al.: Transfer of energy to high frequencies in the cubic defocusing nonlinear Schrodinger equation. Invent. Math. 181(1), 39-113 (2010) 